\title{
Controlling Release From pH-Responsive Microcapsules
}

\author{
Alireza Abbaspourrad, ${ }^{\dagger}$ Sujit S. Datta, ${ }^{\dagger}$ and David A. Weitz* \\ Department of Physics and SEAS, Harvard University, Cambridge, Massachusetts 02138, United States
}

ABSTRACT: We report a microfluidic approach to produce monodisperse $\mathrm{pH}$-responsive microcapsules with precisely controlled release behavior. The solid microcapsule shells are composed of a biocompatible $\mathrm{pH}$-responsive polymer and robustly encapsulate an active material; however, when exposed to a trigger $\mathrm{pH}$, the shells degrade and ultimately release the microcapsule contents. We control the trigger $\mathrm{pH}$ by using polymers that dissolve at different $\mathrm{pH}$ values. We independently control the time at which the microcapsule contents are released by carefully controlling the shell thickness. Moreover, we independently control the rate at which the encapsulated contents are released by making hybrid

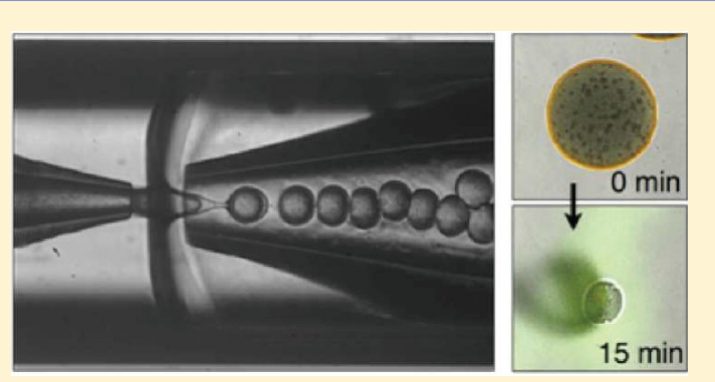
shells composed of a mixture of a $\mathrm{pH}$-responsive polymer and varying proportions of another, solid, $\mathrm{pH}$-unresponsive polymer. This enables us to achieve monodisperse microcapsules that robustly encapsulate an active material, only releasing it when exposed to a desired $\mathrm{pH}$, after a prescribed time delay, and at a prescribed rate.

\section{INTRODUCTION}

Microcapsules are promising candidates for encapsulating, delivering, and controllably releasing many technologically important actives, including agricultural chemicals, ${ }^{1,2}$ surfactants for enhanced oil recovery, cosmetic components, ${ }^{3,4}$ food additives, $^{5-7}$ and pharmaceuticals. ${ }^{8-13}$ These applications often require the microcapsules to release their contents only after exposure to an external stimulus; ${ }^{14-30}$ one important example is a change in $\mathrm{pH}$. Moreover, in many cases, the time delay between exposure and release, as well as the rate of release, must be carefully controlled. Microcapsules that release their contents when exposed to a range of stimuli can be fabricated using a variety of conventional techniques, such as spray drying, $^{31}$ coextrusion, ${ }^{32,33}$ interfacial polymerization, ${ }^{34,35}$ or phase separation. ${ }^{36}$ Unfortunately, the highly variable shear inherent in these approaches typically results in microcapsules with polydisperse sizes and structures, and consequently, poorly controlled encapsulation and release characteristics, even within the same batch. Microfluidic technologies offer a route to overcoming these difficulties and can be used to fabricate a wide variety of monodisperse microcapsules. ${ }^{18,37-42}$ However, controlling the kinetics of release from such microcapsules remains challenging; this severely limits their use in many practical applications.

In this paper, we report a microfluidic approach to produce monodisperse $\mathrm{pH}$-responsive microcapsules with precisely controlled release properties. We use a capillary microfluidic device to prepare water-in-oil-in-water (W/O/W) double emulsion drops as templates; ${ }^{43-48}$ the middle oil phase is a solution of a $\mathrm{pH}$-responsive polymer, which forms a uniform solid shell upon dissolution of the solvent into the outer phase. When exposed to a trigger $\mathrm{pH}$, this shell dissolves at a constant rate, ultimately releasing the microcapsule contents. By using polymers that respond to different $\mathrm{pH}$ values, we fabricate microcapsules that respond to either acidic or basic conditions.
We exploit the exquisite flow control afforded by microfluidics to control the shell thickness and, consequently, the time delay before the contents are released. Moreover, we prepare hybrid microcapsules with shells composed of a mixture of a $\mathrm{pH}$ responsive and a $\mathrm{pH}$-unresponsive polymer; when exposed to a trigger $\mathrm{pH}$, these shells dissolve only at their $\mathrm{pH}$-responsive regions. Thus, by varying the proportions of the different polymers, we independently control the rate at which the microcapsule contents are released. This approach enables us to achieve monodisperse microcapsules that robustly encapsulate their contents, only releasing them when exposed to a desired $\mathrm{pH}$, after a prescribed time delay, and at a prescribed rate.

\section{EXPERIMENTAL DETAILS}

We use a glass capillary microfluidic device to prepare monodisperse $\mathrm{W} / \mathrm{O} / \mathrm{W}$ double emulsion drops as templates to form microcapsules. ${ }^{43}$ The device consists of two tapered cylindrical capillaries inserted into the opposite ends of a square capillary, whose inner dimension is slightly larger than the outer diameter of the cylindrical capillaries, as illustrated by the optical micrograph in Figure 1a. This configuration enables us to accurately align both cylindrical capillaries. We use the left cylindrical capillary to inject the innermost aqueous phase, a $5 \mathrm{wt}$ $\%$ aqueous solution of polyvinyl alcohol (PVA) of molecular weight $13000-23000$, seeded with a small amount of 6-nm-diameter quantum dots for flow visualization. We treat this capillary with $n$ octadecyltrimethoxysilane; this renders its surface hydrophobic, preventing wetting of the aqueous phase on the capillary wall. To fabricate solid microcapsules, we use a middle oil phase composed of a 5-15 wt \% solution of an anionic diblock copolymer of acrylic acid and methyl methacrylate (PAA- $b$-PMMA) in a mixture of $70 \mathrm{vol} \%$ chloroform and 30 vol\% tetrahydrofuran (THF). ${ }^{23}$ We inject this oil from the left, forcing it to flow in the same direction as the inner aqueous phase, through the interstices between the left cylindrical

Received: August 8, 2013

Revised: September 16, 2013

Published: September 16, 2013 

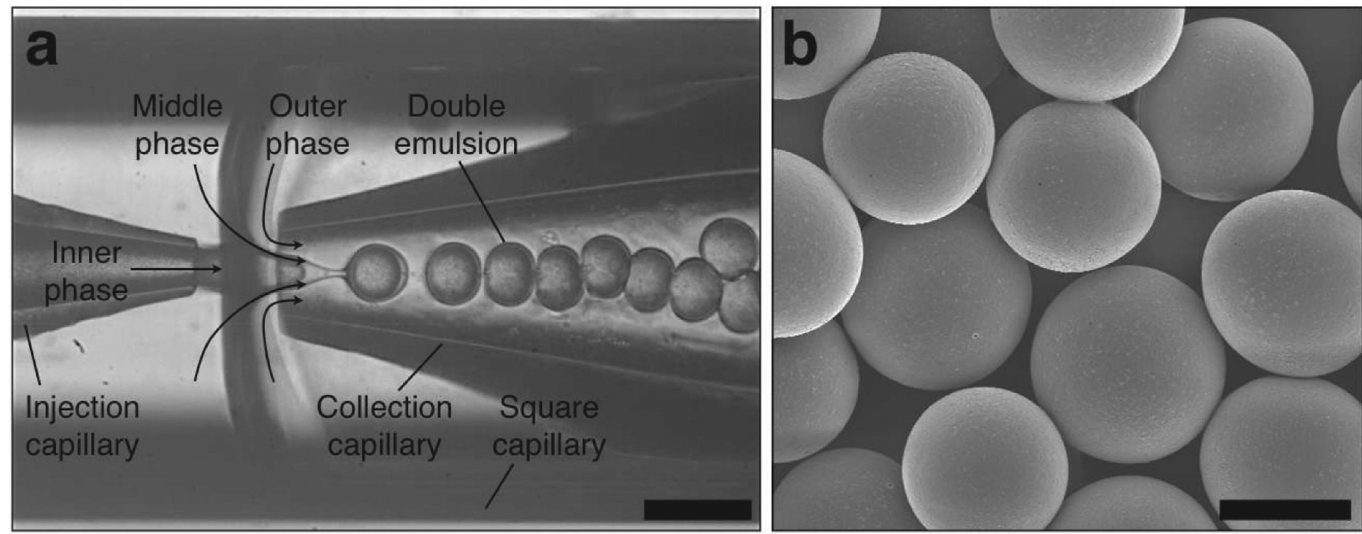

Figure 1. (a) Optical micrograph of a glass capillary microfluidic device as it produces monodisperse double emulsions. Scale bar is $250 \mu \mathrm{m}$. (b) Scanning electron microscope micrograph of monodisperse, uniform, solid microcapsules formed after evaporation of the solvent from the middle phase of the double emulsion drops. Scale bar is $100 \mu \mathrm{m}$.
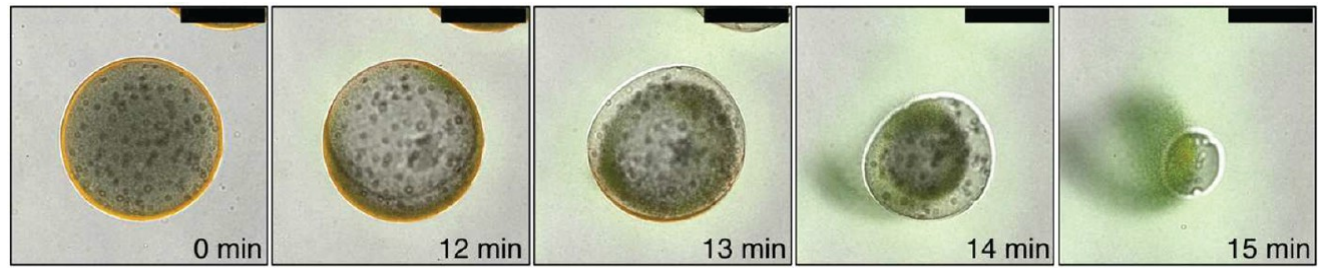

Figure 2. Optical micrographs showing the degradation of the 500-nm-thick shell of a microcapsule, leading to the release of an encapsulated dye starting at $12 \mathrm{~min}$ (green; fluorescence micrograph superimposed). The microcapsules also encapsulate, and subsequently release, polystyrene particles used for enhanced visualization (gray). Scale bar is $100 \mu \mathrm{m}$. Time stamp shows time elapsed after $\mathrm{pH}$ is raised to 9 .
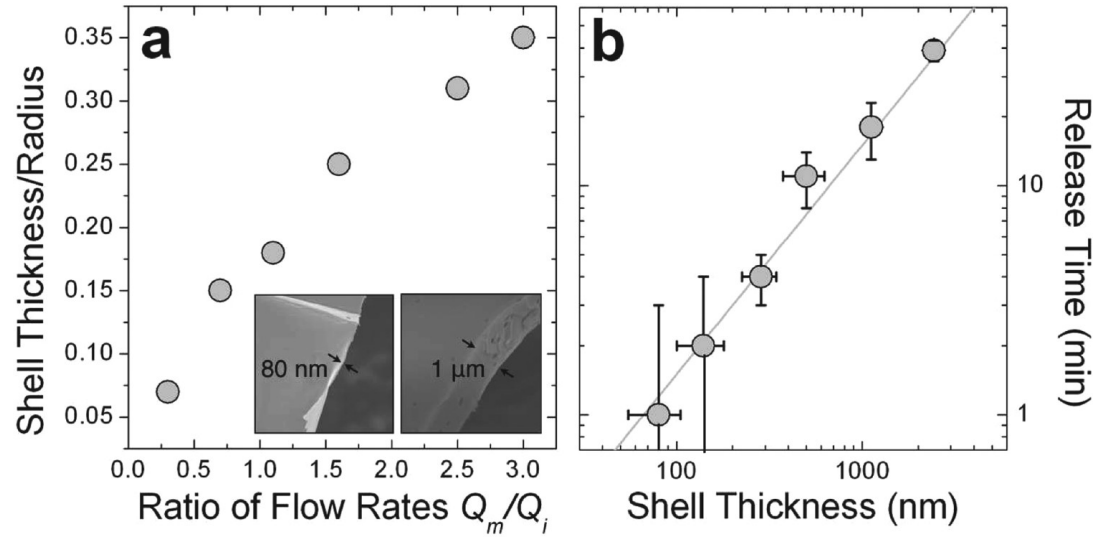

Figure 3. (a) Ratio of microcapsule shell thickness to shell radius, measured using scanning electron microscopy, increases with increasing ratio of middle phase flow rate $Q_{m}$ to inner phase flow rate $Q_{\text {. }}$. Example scanning electron micrographs for thin and thick shells are shown in the inset. (b) Time delay before release begins, quantified using fluorescence microscopy of microcapsules encapsulating a fluorescent dye, increases with increasing shell thickness, for shells triggered at $\mathrm{pH}=9$; gray line shows linear relationship. Vertical error bars show standard deviation in measured release time, while horizontal error bars show standard deviation in shell thickness of each batch.

capillary and the square capillary. The outermost aqueous phase is a 10 wt \% aqueous solution of PVA containing 15 wt \% THF; we inject it from the right, forcing it to flow in the opposite direction, through the interstices between the right cylindrical capillary and the square capillary. We operate this hydrodynamic focusing geometry in the dripping regime, causing the inner and middle phases to break up at the orifice of the right cylindrical capillary; this forms monodisperse $\mathrm{W} / \mathrm{O} / \mathrm{W}$ double emulsions, as shown in Figure 1a.

We use the right cylindrical capillary to collect these double emulsion drops; this capillary is treated with 2-(methoxy(polyethyleneoxy)propyl)trimethoxysilane, rendering its surface hydrophilic and preventing wetting of the middle oil phase on the capillary wall. After the double emulsion drops are collected, the solvent in the middle oil phase slowly diffuses into the outer continuous phase; this forces the PAA- $b$-PMMA to precipitate. Within $1 \mathrm{~h}$, this forms a uniform solid shell of radius $\approx 69 \mu \mathrm{m}$, as exemplified in Figure 1b. We wash the monodisperse microcapsules thus formed with water, adjusted to have $\mathrm{pH}=6$, three times to remove any residual solvent or any surfactant from the continuous phase. We verify that the microcapsule shells are solid by crushing the microcapsules between two glass slides, as well as by imaging the microcapsules directly using scanning electron microscopy. To study the ability of the microcapsules to encapsulate an active material, we encapsulate either 6-nm-diameter quantum dots or a fluorescent polymer (6 kDa FITCdextran), in the microcapsule cores, and measure the change in the fluorescence intensity of the cores over time. After 30 days, we find that only $3 \%$ of the quantum dots leak from the microcapsule cores; by 


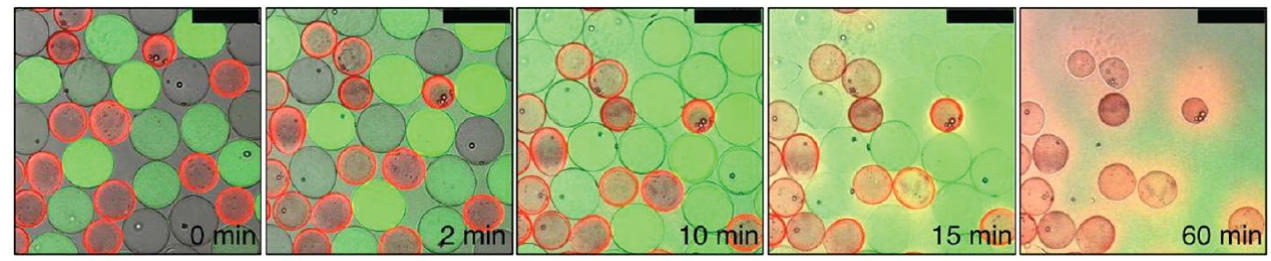

Figure 4. Optical micrographs showing the release, starting at $2 \mathrm{~min}$, of an encapsulated dye from microcapsules with 500-nm-thick shells (green; fluorescence micrograph superimposed). A second encapsulated dye is then released, starting at 15 min, from microcapsules with 4- $\mu \mathrm{m}$-thick shells (red; fluorescence micrograph superimposed). Scale bar is $250 \mu \mathrm{m}$. Time stamp shows time elapsed after $\mathrm{pH}$ is raised to 9 .

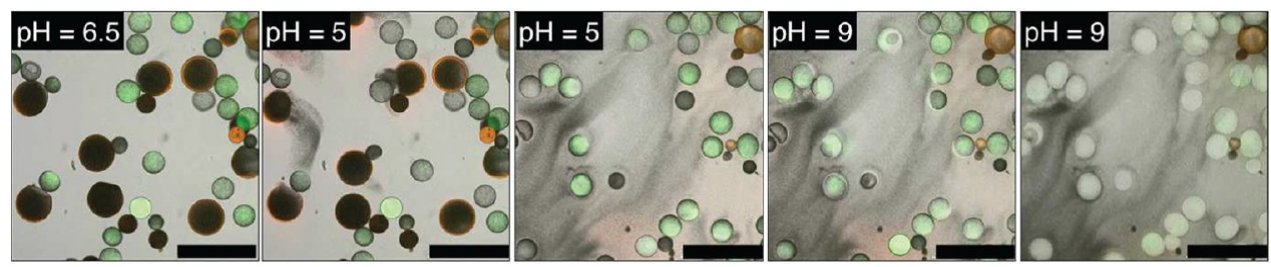

Figure 5. Optical micrographs showing the release of an encapsulated dye only from acid-responsive DMAEMA-BMA-MMA microcapsules (yellow; fluorescence micrograph superimposed) when the $\mathrm{pH}$ is reduced to 5 (second and third frames). A second encapsulated dye (green; fluorescence micrograph superimposed) and $1 \mu \mathrm{m}$ polystyrene particles (gray) are then released from base-responsive PAA- $b$-PMMA microcapsules when the $\mathrm{pH}$ is increased to 9 (fourth and fifth frames). Scale bar is $500 \mu \mathrm{m}$. The shells of both types of capsules are $500 \mathrm{~nm}$ thick.

contrast, we find that $18 \%$ of the smaller FITC-dextran leaks from the microcapsule cores.

\section{RESULTS AND DISCUSSION}

Controlling Release Time. When exposed to a basic $\mathrm{pH}>$ 7, the polymer chains making up the shells become highly charged and repel each other. ${ }^{23}$ This causes the microcapsule shells to dissolve at a constant rate, starting at their exteriors; after a time delay of several minutes, the shells become fully dissolved and the microcapsules release their contents, as shown in Figure 2. We expect microcapsules with thicker shells to take longer to fully dissolve and ultimately release their contents; thus, to control the time of release, we exploit the precise flow control afforded by microfluidics to prepare microcapsules of the same outer radius but with different shell thicknesses. We do this by varying the flow rates of the inner and middle phases, $Q_{i}$ and $Q_{m}$, respectively, while maintaining their sum, and the flow rate of the outer phase, $Q_{0}$, at constant values of $1.2 \mathrm{~mL} / \mathrm{h}$ and $6.0 \mathrm{~mL} / \mathrm{h}$, respectively. Crucially, the thickness of the middle phase of each double emulsion drops, and consequently the thickness of the solid shell formed from it increases with increasing $Q_{m} / Q_{\text {, }}$, as shown in Figure 3a. To quantify the release kinetics for microcapsules of different shell thicknesses, we monitor individual microcapsules using fluorescence microscopy, measuring the time between initiation of a trigger through exposure to a $\mathrm{pH}=9$ and the beginning of the release from their interiors. ${ }^{49}$ The time delay increases linearly with increasing shell thickness, varying from just a minute up to tens of minutes, as shown in Figure $3 \mathrm{~b}$; this is consistent with our expectation. We note that this approach is general, and the release can be triggered by any $\mathrm{pH}$ larger than 7; we demonstrate this concept by choosing a trigger $\mathrm{pH}$ of 9 . The release kinetics may depend on the exact trigger $\mathrm{pH}$ used.

By fabricating and mixing varying amounts of different populations of capsules, each characterized by a different shell thickness, we can precisely control the distribution of release times over a range spanning $1 \mathrm{~min}$ to nearly $1 \mathrm{~h}$. This enables us to program sequential release from different microcapsules; to illustrate this, we fabricate and mix two different populations of microcapsules, having either thin $500 \mathrm{~nm}$ shells or thick 4 $\mu \mathrm{m}$ shells. We distinguish the two populations by encapsulating a green and a red dye within the microcapsules having thin and thick shells, respectively (first frame, Figure 4). We increase the $\mathrm{pH}$ of the continuous phase to 9, forcing the shells to dissolve. After $2 \mathrm{~min}$, the microcapsules with thin shells release their contents; in contrast, the microcapsules with thicker shells continue to stably encapsulate their contents, as shown in the second and third frames of Figure 4. These subsequently release their contents after an additional $13 \mathrm{~min}$ have passed, as shown in the last two frames of Figure 4. These results demonstrate that varying the microcapsule shell thickness provides a straightforward means of programming sequential release at different times.

Controlling Release $\mathrm{pH}$. This experimental approach can be used to trigger release at a range of different $\mathrm{pH}$ conditions. To illustrate its generality, we apply the same microfluidic device but use a different middle oil phase; this enables us to prepare microcapsules that release their contents under acidic conditions. The oil phase is an $8 \mathrm{wt} \%$ solution of a cationic triblock copolymer of poly( $n$-butyl methacrylate-(2-dimethylaminoethyl)-methacrylate-methyl methacrylate) (DMAEMABMA-MMA) in a mixture of $70 \mathrm{vol} \%$ chloroform and $30 \mathrm{vol}$ $\%$ THF. $^{50}$ Similar to the previous case, after the double emulsion drops are formed and collected, the solvent in the middle oil phase slowly diffuses into the continuous phase; this forces the polymer to precipitate, forming a uniform solid shell. We again study the ability of the microcapsules to encapsulate an active material by encapsulating either quantum dots or FITC-dextran in their cores. After 30 days, we find that only $5 \%$ of the quantum dots leak from the microcapsule cores; by contrast, we find that $15 \%$ of the smaller FITC-dextran leaks from the microcapsule cores.

In contrast to the PAA- $b$-PMMA case, the DMAEMA-BMAMMA polymer chains become highly charged only under acidic conditions; consequently, the microcapsule shells thus formed dissolve and release their contents only for $\mathrm{pH}<6 .{ }^{51,52}$ We are therefore able to prepare microcapsules that very stably 
encapsulate their contents and only release them when exposed to either acidic or basic conditions.

This experimental approach enables us to program the sequential release of different actives upon exposure to different $\mathrm{pH}$ conditions; to illustrate this, we fabricate and mix two different populations of acid-responsive and base-responsive microcapsules. We distinguish the two populations by encapsulating a green dye within the base-responsive PAA- $b$ PMMA microcapsules, and a yellow dye, along with $1 \mu \mathrm{m}$ polystyrene particles, within the acid-responsive DMAEMABMA-MMA microcapsules (first frame, Figure 5). Both populations of microcapsules are stable in water adjusted to have $\mathrm{pH}=6.5$. We then decrease the $\mathrm{pH}$ of the continuous phase to 5; while the base-responsive microcapsules remain stable, the acid-responsive microcapsules quickly release their contents, as shown in the second and third frames of Figure 5. We subsequently increase the $\mathrm{pH}$ of the continuous phase to 9; this forces the base-responsive microcapsules to also release their contents, as shown in the last two frames of Figure 5. These observations demonstrate that varying the microcapsule shell composition provides a straightforward means of programming sequential release at different $\mathrm{pH}$. We note that this approach is general, and the release can be triggered by any $\mathrm{pH}$ smaller than 6; we demonstrate this concept by choosing a trigger $\mathrm{pH}$ of 5 . The release kinetics may depend on the exact trigger $\mathrm{pH}$ used.

Controlling Release Rate. The $\mathrm{pH}$-responsive polymers making up the microcapsule shells phase separate when solidified in the presence of another, $\mathrm{pH}$-unresponsive, polymer. We exploit this effect to prepare hybrid microcapsules that, when exposed to a trigger $\mathrm{pH}$, selectively dissolve only at parts of their shells. We again apply our microfluidic approach, using a different middle oil phase; we use a mixture of $0-20 \mathrm{wt}$ $\%$ of the base-responsive PAA- $b$-PMMA with $80-100$ wt \% $\mathrm{pH}$-unresponsive ethylene glycol phenyl ether methacrylate monomer, $0.5 \mathrm{wt} \%$ 1,6- hexanediol dimethacrylate cross-linker, and 0.2 wt $\%$ 2-hydroxy-2-methylpropiophenone photoinitiator. Immediately after the double emulsion drops are formed, they are irradiated with UV light; this photopolymerizes the $\mathrm{pH}$ unresponsive polymer in the middle oil phase. ${ }^{53-56}$ We then collect the drops in water adjusted to have $\mathrm{pH}=6$. Similar to the previous case, the $\mathrm{pH}$-responsive polymer precipitates, completing the formation of a uniform, solid, hybrid shell. We again study the ability of the microcapsules to encapsulate an active material by encapsulating either quantum dots or FITCdextran in their cores. After 30 days, we find no measurable leakage of the quantum dots from the hybrid microcapsule cores; by contrast, we find that only $6-11 \%$ of the smaller FITC-dextran leaks from the hybrid microcapsule cores.

When these hybrid microcapsules are exposed to a trigger $\mathrm{pH}>7$, only the base-responsive portions of their shells dissolve, forming holes; this is illustrated by the schematics and scanning electron micrographs shown in the top panel of Figure 6. This selective dissolution reflects the phase separation between the $\mathrm{pH}$-responsive and $\mathrm{pH}$-unresponsive polymers in the microcapsule shells. The size of the holes formed increases with increasing PAA- $b$-PMMA content, as shown in Figure 6; this presumably increases the permeability of the degraded shell and hence the rate at which it releases its contents. ${ }^{57}$ We test this hypothesis by fabricating a hybrid capsule of varying PAA$b$-PMMA content and use confocal fluorescence microscopy to monitor the dynamics of their release when exposed to a trigger $\mathrm{pH}$. Importantly, we find that the release rate increases with
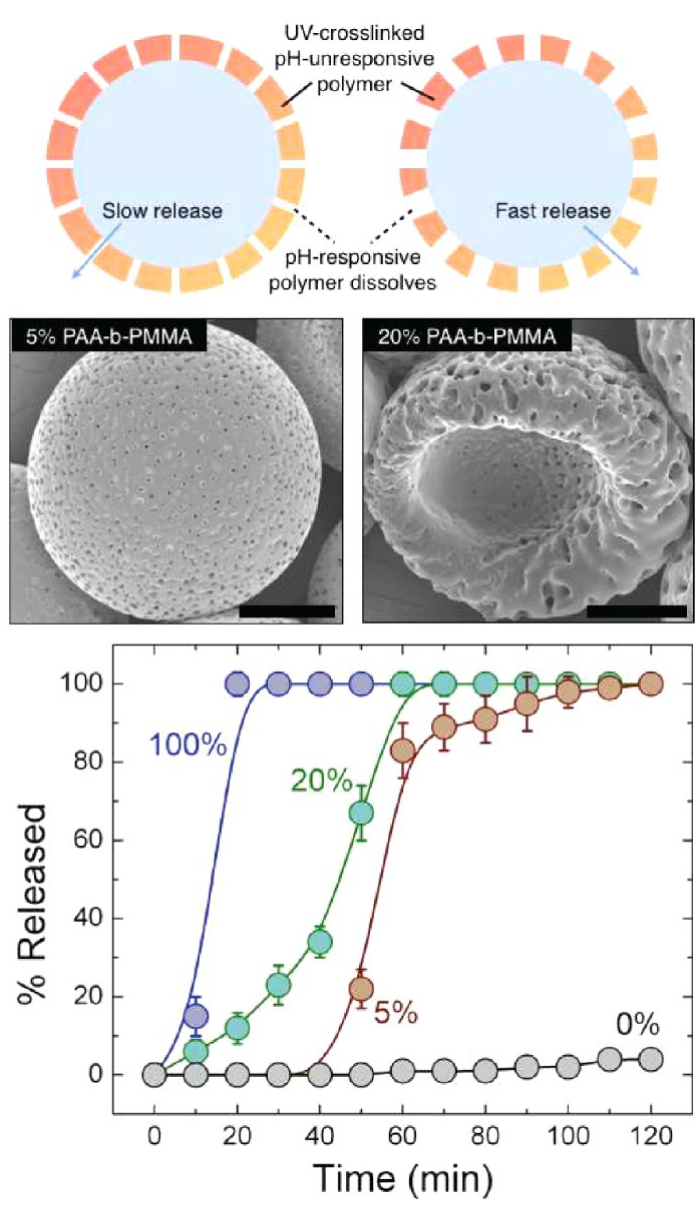

Figure 6. (Top) Schematic representation and (middle) scanning electron microscope micrographs of hybrid microcapsules with holes formed in their shells after degradation in an unfavorable $\mathrm{pH}=8.5$. The size of the holes increases with increasing $\mathrm{pH}$-responsive PAA- $b$ PMMA content in the shell. Scale bars are $80 \mu \mathrm{m}$. Shell thickness is 2.4 $\mu \mathrm{m}$ for both microcapsules. (Bottom) Amount of encapsulated material released, quantified by monitoring the relative intensity in the core of microcapsules encapsulating a fluorescent dye, increases with time; capsules are triggered at $\mathrm{pH}=9$. The rate at which the material is released increases with the PAA- $b$-PMMA content of the shell (numbers next to each curve). Vertical error bars show standard deviation of measured intensity in each batch, at each time point.

increasing PAA- $b$-PMMA content, as shown by the different curves in Figure 6. These observations confirm that the microcapsule release rate can be independently tuned by the composition of the hybrid shell.

\section{CONCLUSIONS}

In this work, we report a microfluidic approach to produce double emulsion-templated monodisperse $\mathrm{pH}$-responsive microcapsules with precisely controlled release properties. By controlling the shell thickness, we control the amount of time required to fully dissolve the shell and hence the time at which the microcapsule contents are ultimately released. By using different $\mathrm{pH}$-responsive polymers to form the microcapsule shell, we control the trigger $\mathrm{pH}$. This can range from highly acidic conditions, such as those encountered in the human stomach, or basic conditions, such as those encountered in parts of the small intestine and the colon. ${ }^{58}$ The $\mathrm{pH}$-responsive polymers we use are enteric and biocompatible; thus, our microcapsules may be promising candidates for the delivery of 
pharmaceutical products to different areas of the human digestive system. Moreover, by varying the fraction of the microcapsule shell made up of $\mathrm{pH}$-responsive polymer, we independently control the shell permeability and hence the rate at which each microcapsule releases its contents. This approach is general and can be applied to a wide range of polymers. We note that the $\mathrm{pH}$-unresponsive polymer we use here is not biocompatible; further work is therefore required to develop fully biocompatible hybrid microcapsules.

\section{AUTHOR INFORMATION}

\section{Corresponding Author}

*E-mail: weitz@seas.harvard.edu.

\section{Author Contributions}

${ }^{\dagger}$ These authors contributed equally to this work.

\section{Notes}

The authors declare no competing financial interest.

\section{ACKNOWLEDGMENTS}

This work was supported by the NSF (DMR-1006546), the Harvard MRSEC (DMR- 0820484), and the Advanced Energy Consortium (http://www.beg.utexas.edu/aec/), whose member companies include BP America Inc., BG Group, Petrobras, Schlumberger, Shell, and Total. S.S.D. acknowledges funding from ConocoPhillips. A.A. acknowledges Evonik Industries for gifting the PAA- $b$-PMMA and DMAEMA-BMA-MMA polymers.

\section{REFERENCES}

(1) Friedman, S. P.; Mualem, Y. Diffusion of fertilizers from controlled release sources, uniformly distributed in soil. Fert. Res. 1994, 39, 19-30.

(2) Drake, C. F. Controlled delivery agricultural capsule and method of making. United States Patent 479474, 1998,

(3) Lotan, T.; Eckhouse, S. Methods compositions and devices utilizing stinging cells/capsules for delivering a therapeutic or a cosmetic agent into a tissue. United States Patent 7338665, 2008,

(4) Lapidot, N.; Gans, O.; Biagini, F.; Sosonkin, L.; Rottman, C. Advanced Sunscreens: UV absorbers encapsulated in sol-gel glass microcapsules. J. Sol-Gel Sci. Technol. 2003, 26, 67-72.

(5) Faria, A. F.; Mignone, R. A.; Montenegro, M. A.; Mercadante, A. Z.; Borsarelli, C. D. Characterization and singlet oxygen quenching capacity of spray-dried microcapsules of edible biopolymers containing antioxidant molecules. J. Agric. Food Chem. 2010, 58, 8004-8011.

(6) Yeo, Y.; Bellas, E.; Firestone, W.; Langer, R.; Kohane, D. S. Complex coacervates for thermally sensitive controlled release of flavor compounds. J. Agric. Food Chem. 2005, 53, 7518-7525.

(7) Risch, S. J., Reineccius, G. A., Eds. Encapsulation and controlled release of food ingredients; American Chemical Society: Washington, DC, 1995.

(8) Ichikawa, H.; Fujioka, K.; Adeyey, M. C.; Fukumori, Y. Use of ion-exchange resins to prepare $100 \mu \mathrm{m}$-sized microcapsules with prolonged drug-release by the Wurster process. Int. J. Pharm. 2001, 216, 67-76.

(9) Hu, S. H.; Tsai, C. H.; Liao, C. F.; Liu, D. M.; Chen, S. Y. Controlled rupture of magnetic polyelectrolyte microcapsules for drug delivery. Langmuir 2008, 24, 11811-11818.

(10) Zhao, Q.; Han, B.; Wang, Z.; Gao, C.; Peng, C.; Shen, J. Hollow chitosan-alginate multilayer microcapsules as drug delivery vehicle: Doxorubicin loading and in vitro and in vivo studies. Nanomedicine 2007, 3, 63-74.

(11) He, W.; Gu, X.; Liu, S. Surfactant-free one step synthesis of dual-functional polyurea microcapsule: Contact infection control and drug delivery. Adv. Funct. Mater. 2012, 22, 4023-4031.
(12) Lensen, D.; Gelderblom, E. C.; Vriezema, D. M.; Marmottant, P.; Verdonschot, N.; Versluis, M.; Jong, N.; Hest, J. C. M. Biodegradable polymeric microcapsules for selective ultrasoundtriggered drug release. Soft Matter 2011, 7, 5417-5422.

(13) Taboada, A. D.; Maillet, L.; Banoub, J. H.; Lorch, M.; Rigby, A. S.; Boa, A. N.; Atkin, S. L.; Mackenzie, G. Protein free microcapsules obtained from plant spores as a model for drug delivery: Ibuprofen encapsulation, release and taste masking. J. Mater. Chem. B 2013, 1, $707-713$.

(14) Pastine, S. J.; Okawa, D.; Zettl, A.; Frechet, J. M. Chemicals on demand with phototriggerable microcapsules. J. Am. Chem. Soc. 2009, 131, 13586.

(15) Geest, B. G. D.; McShane, M. J.; Demeester, J.; Smedt, S. C. D.; Hennink, W. E. Microcapsules ejecting nanosized species into the environment. J. Am. Chem. Soc. 2008, 130, 14480.

(16) Mauser, T.; Dejugnat, C.; Mohwald, H.; Sukhurov, G. B. Microcapsules made of weak polyelectrolytes: Templating and stimuliresponsive properties. Langmuir 2006, 22, 5888.

(17) Shchukin, D. G.; Kohler, K.; Mohwald, H. Microcontainers with electrochemically reversible permeability. J. Am. Chem. Soc. 2006, 129, 4560.

(18) Zhang, H.; Tumarkin, E.; Peerani, R.; Nie, Z.; Sullan, R. M. A.; Walker, G. C.; Kumacheva, E. Microfluidic production of biopolymer microcapsules with controlled morphology. J. Am. Chem. Soc. 2006, $128,12205$.

(19) Ye, C.; Chen, A.; Colombo, P.; Martinez, C. Ceramic microparticles and micro-capsules via microfluidic processing of a preceramic polymer. J. R. Soc., Interface 2010, 7, S461.

(20) Chen, R.; Pearce, D. J. G.; Fortuna, S.; Cheung, D. L.; Bon, S. A. Polymer vesicles with a colloidal armor of nanoparticles. J. Am. Chem. Soc. 2011, 133, 2151.

(21) Huang, X.; Appelhans, D.; Formanek, P.; Simon, F.; Voit, B. Tailored synthesis of intelligent polymer nanocapsules: An investigation of controlled permeability and $\mathrm{pH}$-dependent degradability. ACS Nano 2012, 6, 9718-9726.

(22) Narita, T.; Takakura, H.; Ogata, N.; Kawakita, H.; Oishi, Y. Selfpulsation observed in $\mathrm{pH}$-sensitive microcapsules. Chem. Commun. 2013, 49, 919-921.

(23) Miguel, A. S.; Scrimgeour, J.; Curtis, J. E.; Behrens, S. H. Smart colloidosomes with a dissolution trigger. Soft Matter 2010, 6, 31633166.

(24) Kozlovskaya, V.; Wang, Y.; Higgins, W.; Chen, J.; Chen, Y.; Kharlampieva, E. pH-Triggered shape response of cubical ultrathin hydrogel capsules. Soft Matter 2012, 8, 9828-9839.

(25) Du, J.; Fan, L.; Liu, Q. pH-Sensitive block copolymer vesicles with variable trigger points for drug delivery. Macromolecules 2012, 45, $8275-8283$.

(26) Gil, P. R.; Nazarenus, M.; Ashraf, S.; Parak, W. J. pH-Sensitive capsules as intracellular optical reporters for monitoring lysosomal $\mathrm{pH}$ changes upon stimulation. Small 2012, 8, 943-948.

(27) Felber, A. E.; Dufresne, M. H.; Leroux, J. C. pH-Sensitive vesicles, polymeric micelles, and nanospheres prepared with polycarboxylates. Adv. Drug Delivery Rev. 2012, 64, 979-992.

(28) Hu, H.; Wang, H.; Du, Q. Preparation of $\mathrm{pH}$-sensitive polyacrylic acid hollow microspheres and their release properties. Soft Matter 2012, 8, 6816.

(29) Hamad, S. A.; Stoyanov, S. D.; Paunov, V. N. Triggered cell release from shellac-cell composite microcapsules. Soft Matter 2012, 8, 5069.

(30) Cuomo, F.; Lopez, F.; Ceglie, A.; Maiuro, L.; Miguel, M. G.; Lindman, B. pH-Responsive liposome-templated polyelectrolyte nanocapsules. Soft Matter 2012, 8, 4415.

(31) Wang, Y.; Ye, H.; Zhou, C.; Lv, F.; Bie, X.; Lu, Z. Study on the spray-drying encapsulation of lutein in the porous starch and gelatin mixture. Eur. Food Res. Technol. 2012, 234, 157-163.

(32) Shilpa, A.; Agrawal, S. S.; Ray, A. R. J. Controlled delivery of drugs from alginate matrix. J. Macromol. Sci., Polym. Rev. 2003, 43, $187-221$ 
(33) Tan, C. S.; Jejurikar, A.; Rai, B.; Bostrom, T.; Lawrie, G.; Grohndahl, K. Encapsulation of a glycosaminoglycan in hydroxyapatite/alginate capsules. J. Biomed. Mater. Res. A 2009, 91A, 866-877.

(34) Wang, L. L.; Wang, Z.; Zhang, B. H.; Xiao, K. F. Screening of additives for $1 \%$ abamectin microcapsules suspension. Adv. Mater. Res. 2013, 634, 1090-1094.

(35) Kang, J. H.; Reichmanis, E. Low-threshold photon upconversion capsules obtained by photoinduced interfacial polymerization. Angew. Chem. 2012, 124, 12011-12014.

(36) Yang, R.; Zhang, Y.; Wang, X.; Zhang, Y.; Zhang, Q. Preparation of $n$-tetradecane-containing microcapsules with different shell materials by phase separation method. Sol. Energy Mater. Sol. Cells 2009, 93, 817-822.

(37) Tu, F.; Lee, D. Controlling the stability and size of double emulsion-templated poly(lactic-co-glycolic)acid microcapsules. Langmuir 2012, 28, 9944-9952.

(38) Zhang, M. J.; Wang, W.; Xie, R.; Ju, X. J.; Liu, J.; Gu, Y. Y.; Chu, L. Y. Microfluidic fabrication of monodisperse microcapsules for glucose-response at physiological temperature. Soft Matter 2013, 9, 4150 .

(39) Wei, J.; Ju, X. J.; Xie, R.; Mou, C. L.; Lin, X.; Chu, L. Y. Novel cationic $\mathrm{pH}$-responsive poly( $\mathrm{N}, \mathrm{N}$-dimethylaminoethyl methacrylate) microcapsules prepared by a microfluidic technique. J. Colloid Interface Sci. 2011, 357, 101-108.

(40) Liu, L.; Yang, J. P.; Ju, X. J.; Xie, R.; Liu, Y. M.; Wang, W.; Zhang, J. J.; Niu, C. H.; Chu, L. Y. Monodisperse core-shell chitosan microcapsules for $\mathrm{pH}$-responsive burst release of hydrophobic drugs. Soft Matter 2011, 7, 4821-4827.

(41) Mendes, A. C.; Baran, E. T.; Lisboa, P.; Reis, R. L.; Azevedo, H. $\mathrm{S}$. Microfluidic fabrication of self-assembled peptide-polysaccharide microcapsules as $3 \mathrm{D}$ environments for cell culture. Biomacromolecules 2012, 13, 4039-4048.

(42) Hassan, N.; Cabuil, V.; Abou-Hassan, A. Assembling magnetoplasmonic microcapsules using a microfluidic device. Chem. Commun. 2013, 49, 412-414.

(43) Utada, A. S.; Lorenceau, E.; Link, D. R.; Kaplan, P. D.; Stone, H. A.; Weitz, D. A. Monodisperse double emulsions generated from a microcapillary device. Science 2005, 308, 537-541.

(44) Xu, S.; Nie, Z.; Seo, M.; Lewis, P.; Kumacheva, E.; Stone, H. A.; Garstecki, P.; Weibel, D. B.; Gitlin, I.; Whitesides, G. M. Generation of monodisperse particles by using microfluidics: Control over size, shape, and composition. Angew. Chem. 2005, 117, 7340738.

(45) Nie, Z.; Xu, S.; Se, M.; Lewis, P. C.; Kumacheva, E. Polymer particles with various shapes and morphologies produced in continuous microfluidic reactors. J. Am. Chem. Soc. 2005, 127, 8058-8063.

(46) Tu, F.; Lee, D. Controlling the stability and size of double emulsion-templated poly(lactic-co-glycolic)acid microcapsules. Langmuir 2012, 28, 9944-9952.

(47) Lee, M. H.; Hribar, K. C.; Brugarolas, T.; Kamat, N. P.; Burdick, J. A.; Lee, D. Harnessing interfacial phenomena to program the release properties of hollow microcapsules. Adv. Funct. Mater. 2012, 22, 131138.

(48) Shum, H. C.; Kim, J. W.; Weitz, D. A. Microfluidic fabrication of monodisperse biocompatible and biodegradable polymersomes with controlled permeability. J. Am. Chem. Soc. 2008, 130, 9543-9549.

(49) Datta, S. S.; Shum, H. C.; Weitz, D. A. Controlled buckling and crumpling of nanoparticle-coated droplets. Langmuir 2010, 26, 18612-18616.

(50) Eisele, J.; Haynes, G.; Rosamilia, T. Characterisation and toxicological behaviour of basic methacrylate copolymer for GRAS evaluation. Regul. Toxicol. Pharmacol. 2011, 61, 32-43.

(51) Moustafine, R. I.; Zaharov, I. M.; Kemenova, V. A. Physicochemical characterization and drug release properties of Eudragit E PO/Eudragit L 100-55 interpolyelectrolyte complexes. Eur. J. Pharm. Biopharm. 2006, 63, 26-36.

(52) Liu, H.; Wang, P.; Zhang, X.; Shen, F.; Gogos, C. G. Effects of extrusion process parameters on the dissolution behavior of indomethacin in Eudragit E PO solid dispersions. Int. J. Pharm. 2010, 383, 161-169.

(53) Datta, S. S.; Kim, S. H.; Paulose, J.; Abbaspourrad, A.; Nelson, D. R.; Weitz, D. A. Delayed buckling and guided folding of inhomogeneous capsules. Phys. Rev. Lett. 2012, 109, 134302.

(54) Kim, S. H.; Abbaspourrad, A.; Weitz, D. A. Amphiphilic crescent-moon-shaped microparticles formed by selective adsorption of colloids. J. Am. Chem. Soc. 2011, 133, 5516-5524.

(55) Kim, S. H.; Jeon, S. J.; Yang, S. M. Optofluidic encapsulation of crystalline colloidal arrays with spherical membranes. J. Am. Chem. Soc. 2008, 130, 6040-6046.

(56) Wu, B.; Gong, H. Q. Formation of fully closed microcapsules as microsensors by microfluidic double emulsion. Microfluid. Nanofluid. 2013, 14, 637-644.

(57) Wang, X.; Wang, Q.; Liu, P.; Zhang, X. Construction and characterization of three-dimensionally ordered macroporous polystyrene equipped with $\mathrm{pH}$-responsive switchable windows. Soft Matter 2012, 8, 8847.

(58) Schellekens, R. C. A.; Baltink, J. H.; Woesthuis, E. M.; Stellaard, F.; Kosterink, J. G. W.; Woerdenbag, H. J.; Frijlink, H. W. Film coated tablets (ColoPulse technology) for targeted delivery in the lower intestinal tract: Influence of the core composition on release characteristics. Pharm. Dev. Technol. 2012, 17, 40-47. 